\title{
Modeling and Design of Model predictive controller for flow control loop
}

\author{
Anil.B. Kadu \\ Department of Instrumentation and control Engineering \\ Vishwakarma Institute of Technology, Pune, Maharashtra, India
}

\begin{abstract}
This paper presents Modeling and design of Model predictive control for laboratory experiment closed loop Flow control loop. Model predictive control uses a mathematical model to simulate a process. The system identification tool is used to find out the model of the system. This model then fits the inputs to predict the system behavior. To obtain the model here we are using Data Acquisition card of NI 9862. The main aim of this paper is to build the Model predictive control (MPC) strategy, analyze and compare the control effects with Proportional-Integral-Derivative (PID) control strategy in maintaining a flow control system. And performance of MPC and PID Controller are compared using time domain specifications.
\end{abstract}

Keywords: Data Acquisition card, Model predetive control, Flow control loop, MATLAB7.5, FODPT (First Order plus Delay Time).

\section{INTRODUCTION}

Model Predictive Control, or MPC, is an advanced method of process control that has been in use in the process industries such as chemical plants and oil refineries. Model predictive controllers rely on dynamic models of the process, most often linear empirical models obtained by system identification. Model predictive control (MPC) refers to a class of computer control algorithms that utilize an explicit process model to predict the future response of a plant. At each control interval an MPC algorithm attempts to optimize future plant behavior by computing a sequence of future manipulated variable adjustments. The first input in the optimal sequence is then sent into the plant, and the entire calculation is repeated at subsequent control intervals. Due to the fast development of process industry, the requirements of higher product quality, better product function, and quicker adjustments to the market change have become much stronger, which lead to a demand of a very successful controller design strategy, both in theory and practice. As a closed loop optimal control method based on the explicit use of a process model, model predictive control has proven to be a very effective controller design strategy over the last twenty five years and has been widely used in Process industry such as oil refining, chemical engineering and metallurgy.[4]

\section{EXPERIMENTAL SETUP}

The laboratory set up for this system consists of a tank, a water reservoir, pump, rotameter, a Turbine type flow transmitter, an electro pneumatic converter (I/P converter), a pneumatic control valve, an interfacing module and a Personal Computer (PC). The flow transmitter output is interfaced with computer using Data Acquisition card NI 9812. This module supports 8 analog input and 4 analog output channels with the voltage range of \pm 10 volt. The sampling rate of the module is 18 samples per sec and baud rate is 9600 bytes per sec with 16-bit resolution. Table I shows the technical specifications of the setup.

\begin{tabular}{|c|c|}
\hline Part name & Detials \\
\hline Sump tank & Stainless Steel \\
& Capacity: 30 liter \\
\hline Rotameter & Range (100 - 1000) LPH \\
\hline Pump & Centrifugal 0.5 HP \\
\hline Air Regulater & $0-10 \mathrm{~kg} / \mathrm{cm} 2$ with pressure gauge. \\
\hline
\end{tabular}




\begin{tabular}{|c|c|}
\hline E/P converter & Input (4-20) mA, Output (3 - 15) psi \\
\hline Control Valve & $\begin{array}{c}\text { Size 1/“" Pneumatic actuated, } \\
\text { Type: Air to close Input (3 - 15) psi }\end{array}$ \\
\hline Flow meter & $\begin{array}{l}\text { Turbine type. Range: 0-600 LPH } \\
\text { Output: 4-20 MaType: 3 wireSupply: 24V DC supply. }\end{array}$ \\
\hline
\end{tabular}

\section{Flow control loop setup:}

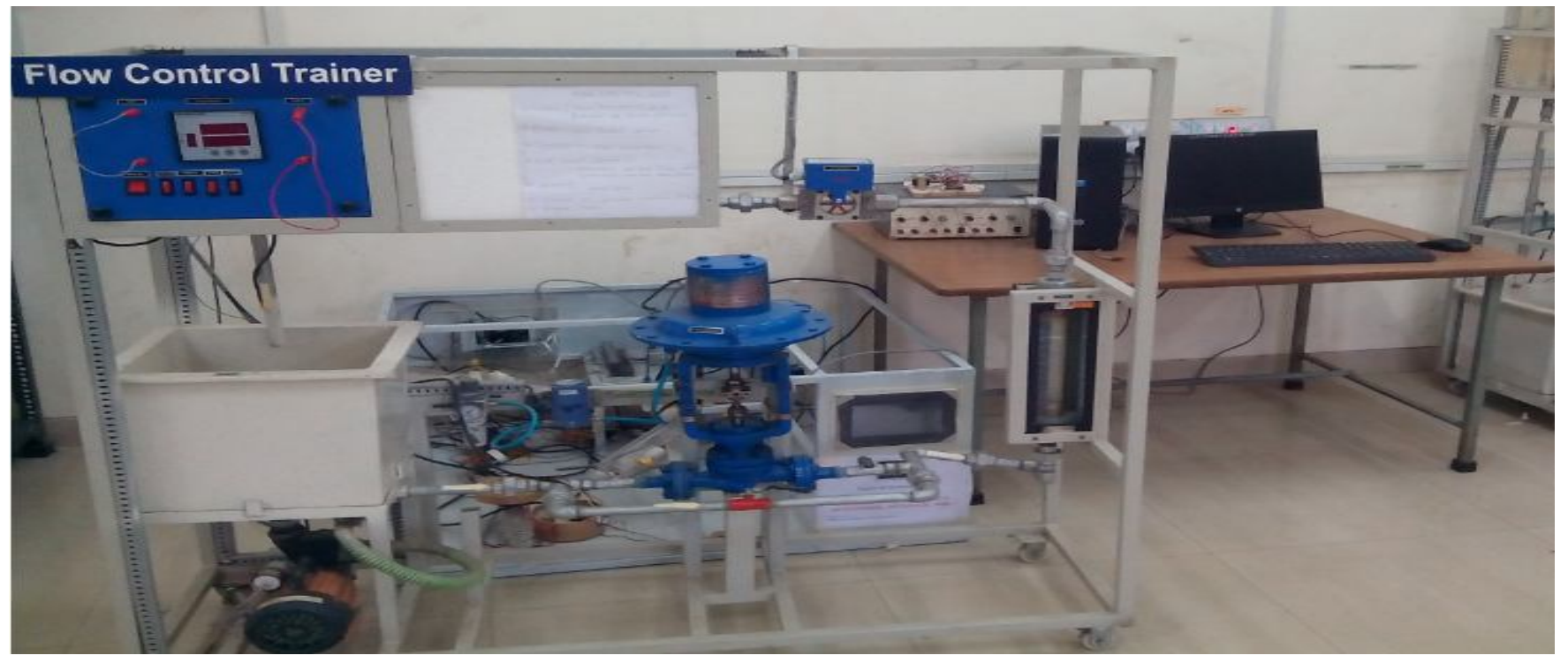

\section{III.MODELING OF SYSTEM USING SYSTEM IDENTIFICATION TOOLBOX:}

Mathematical model of the process is obtained by means of input-output process signals. The advantage in using experiments is that they do not require detailed specific knowledge of researched system, and simple mathematical models that describe the system can be given as a result. The experimental setup of flow control loop and its representation are shown in fig 1. An empirical model of the flow control loop is obtained from the open loop data collecting by exciting the system with step signal. The system is interfaced with personal computer using NI 6012 data acquisition card. The input is the voltage signal applied to the pneumatic valve through the voltage to current converter and it is given to the current to pressure converter $(\mathrm{E} / \mathrm{P})$. The flow of water is measured using turbine flow transmitter having range (4-20) mA corresponding to (100-1000) lph. Again current to voltage converter is used to get the corresponding voltage signal in the range of 1.-5 V.The voltage signal as recorded as output data. The input and output data are recorded with sampling period of $2 \mathrm{~s}$ is used for model identification. The model of the flow system together with components such as valve, converter, flow transmitter, pump and Rotameter is obtained by using the data. The experimental data are approximated to be a FOPDT model and the FOPDT model is represented by

$$
\frac{K_{p} e^{-t_{d} s}}{1+\tau S}
$$

Where

$$
\begin{array}{cl}
K_{P} & \text { is the steady state gain of the system } \\
\tau & \text { is time constant of system } \\
t_{d} & \text { is the dead time of system }
\end{array}
$$




\section{International Journal of Innovations in Engineering and Technology (IJIET) \\ http://dx.doi.org/10.21172/ijiet.83.003}

In the given system, from the open loop response of the Flow Control System, it is seen that by measuring input output data we can create the mathematical models of dynamic systems from measured input-output data by using system Identification Toolbox in MATLAB. The following estimate of the plant is obtained by using system Identification Toolbox as shown in fig 2 and fig 3

Respectively.

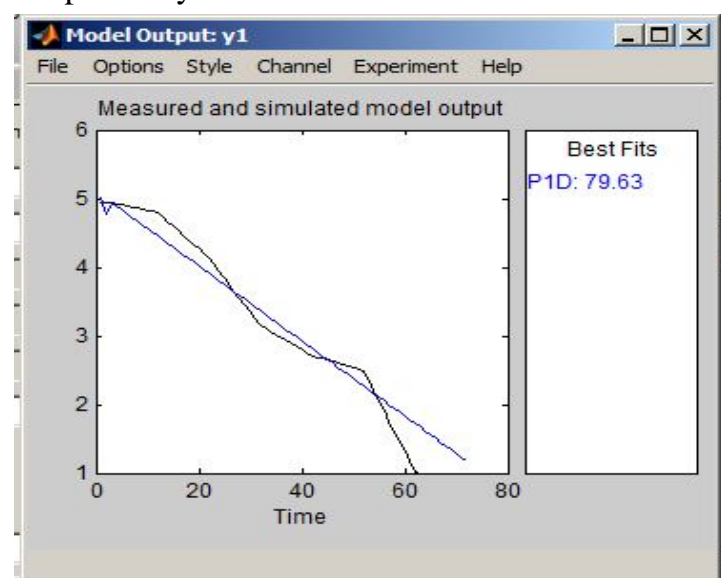

fig 2 Model output dialog box

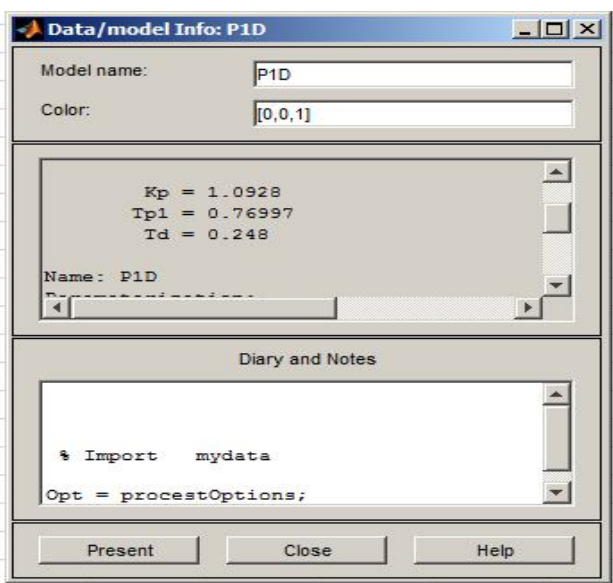

fig 3 Parameters of FOPDT Model

Hence, for the best fit of $79.63 \%$ shown above in figure 4 we get FOPDT model as,

Where

$$
G p(s)=\frac{1.0928 e^{-0.248 s}}{1+0.7699 s}
$$

$$
\begin{aligned}
& K_{P}=1.0928 \text { is the steady state gain of the system } \\
& \tau_{P 1}=0.7699 \quad \text { is time constant of system } \\
& t_{d}=0.248 \quad \text { is the dead time of system }
\end{aligned}
$$

\section{DESIGN OF MODEL PREDICTIVE CONTROLLER}

As briefly discussed in the introduction, MPC controllers are widely applied over process and oil/petrochemical industries due to its capability to deal in an optimal form with input/output process constraints. The model predictive controller uses the models and current plant measurements to calculate future moves in the independent variables that will result in operation that honors' all independent and dependent variable constraints. The MPC then sends this set of independent variable moves to the corresponding regulatory controller set points to be implemented in the process. [11]

\subsection{Model Predictive Control strategy}

Model predictive control (MPC) includes a class of control algorithms that utilize an explicit process model to predict the future response of a plant. At each control interval an MPC algorithm attempts to optimize future plant behavior by computing a sequence of future manipulated variable adjustments. The first input in the optimal sequence is then sent into the plant, and the entire calculation is repeated at subsequent control intervals. The following is a figure 4 shows the basic idea of predictive control based on a single-input, single output plant. 


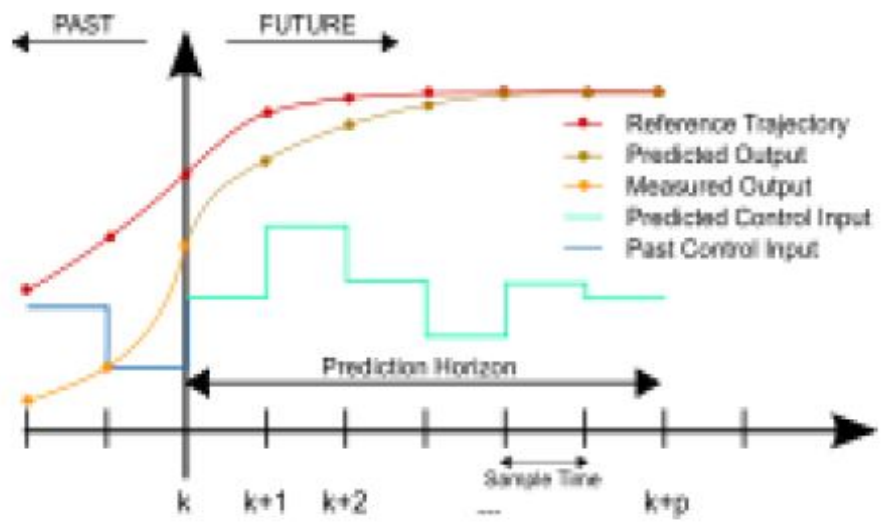

Fig 4 Model Predictive Control Strategy

MPC is based on iterative, finite-horizon optimization of a plant model. At time $t$ the current plant state is sampled and a cost minimizing control strategy is computed (via a numerical minimization algorithm) for a relatively short time horizon in the future: $t, t+T$ Specifically, an online or on-the-fly calculation is used to explore state trajectories that emanate from the current state and find a cost-minimizing control strategy until time. $t+T$ Only the first step of the control strategy is implemented, then the plant state is sampled again and the calculations are repeated starting from the new current state, yielding a new control and new predicted state path. The prediction horizon keeps being shifted forward and for this reason MPC is also called receding horizon control. Although this approach is not optimal, in practice it has given very good results.

\subsection{SISO System: Simulation Example}

\section{SIMULATION RESULTS AND DISCUSSION}

A simple SISO system is taken here for the study - FOPDT system. The objective is to control Flow of system by manipulating water flow rate through valve opening and closing, thus consisting on a 1x1 system; furthermore, both servo (flow control setpoint change) and regulation problems (disturbance rejection) are able to be performed using MPC. But here we have implemented for servo problem (set point tracking purpose).The response of the control strategies (PID and MPC) used to operate the control valve. The transfer function model which relates MV and CV is a first-order plus delay type .The response of the control strategies (PID and MPC) used to operate the control valve.

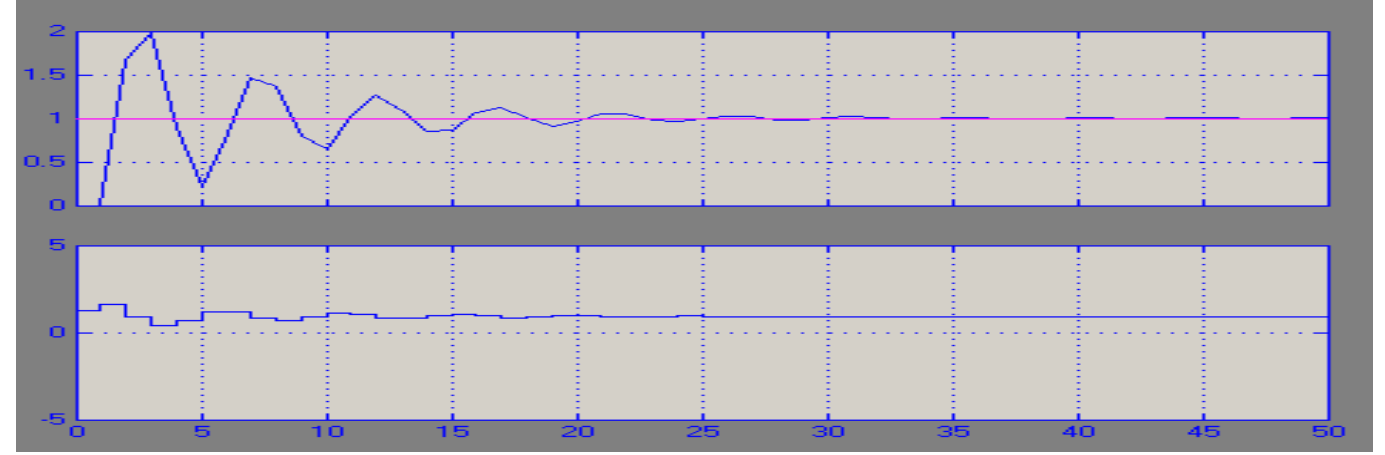

Fig 5 shows unstable response due to weight parameter high.

It is clear that from the fig 5 the system became unstable with such parameters by being too fast due to the Weight parameter value is 0.8 or greater than 0.8.To turn the system slower and more robust, we have to adjust the Weight Now set its value to 0.4 (or lower values).Next fig shows response change when weight parameter change. by a simple change over the speed of the system now a stable yet sluggish closed-loop response is obtained.[11] 


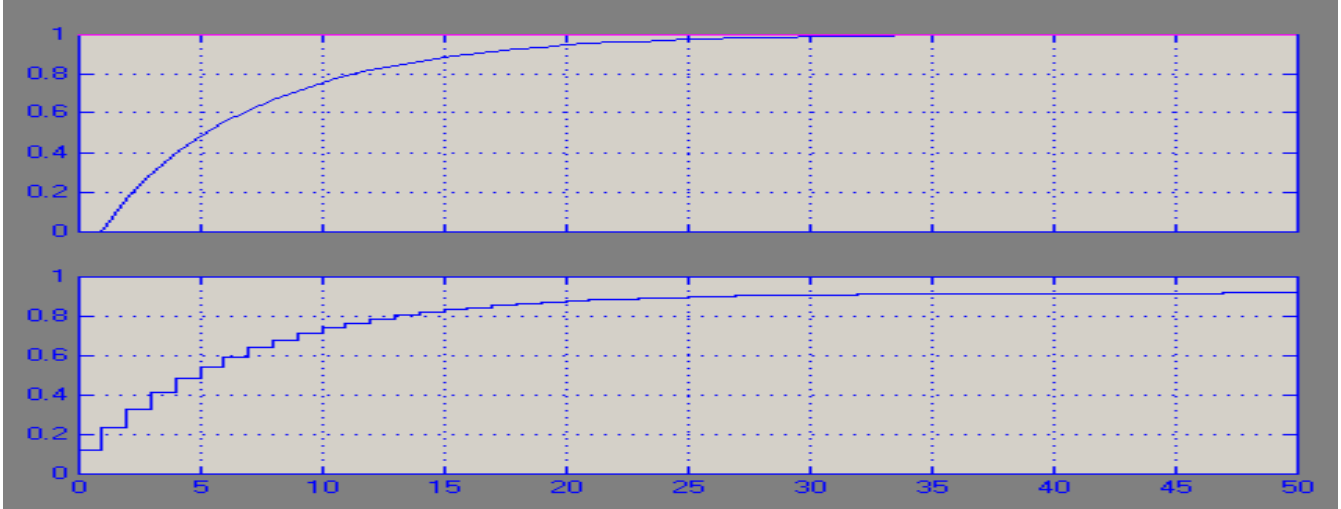

Fig 6 shows stable response due to weight parameter low.

\subsection{Effect of prediction horizon on servo problem:}

It is possible to turn the system response faster by adjusting accordingly the value for prediction horizon. Let us try several different values and choose one which fits a middle ground between robustness and speed as shown in fig $7,8,9$ respectively.

\begin{tabular}{|c|c|c|c|}
\hline Sr.No & Prediction horizon & Control horizon & Settling time \\
\hline 1 & 5 & 1 & 7 Seconds \\
\hline 2 & 10 & 1 & 10 Seconds \\
\hline 3 & 15 & 1 & 8 Seconds \\
\hline
\end{tabular}

$\mathrm{P}=5$ (Prediction horizon) and $\mathrm{M}=1$

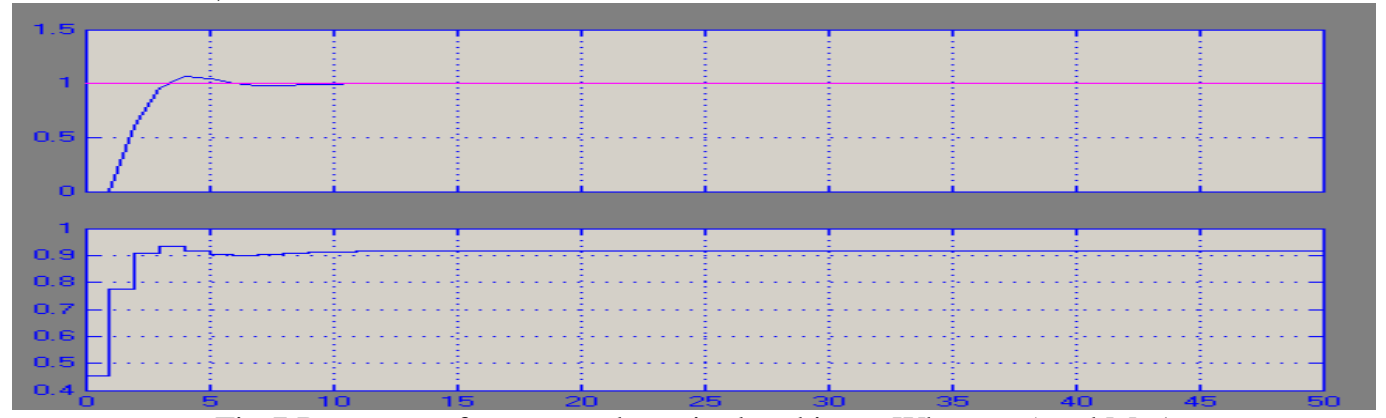

Fig 7 Response of output and manipulated input When $\mathrm{p}=5$ and $\mathrm{M}=1$

$\mathrm{P}=10$ (Prediction horizon) and $\mathrm{M}=1$

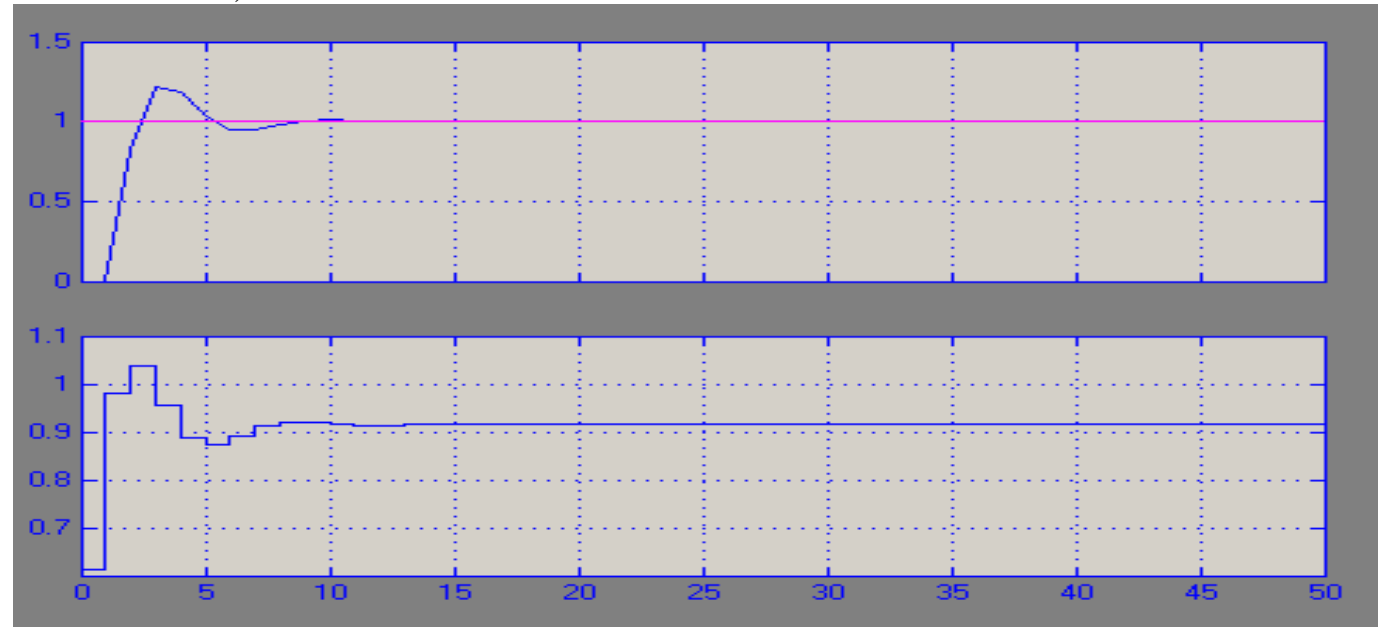

Fig 8 Response of output and manipulated input when $\mathrm{p}=10$ and $\mathrm{M=1}$ 
$\mathrm{P}=15$ (Prediction horizon) and $\mathrm{M}=1$

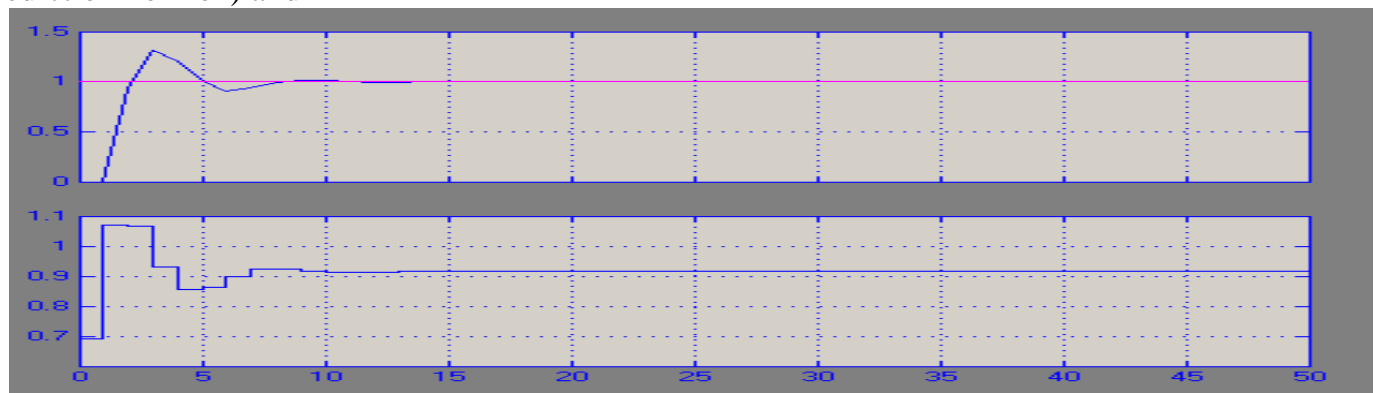

Fig 9 Response of output and manipulated input with $\mathrm{P}=15$ and $\mathrm{M}=1$

If the control horizon $\mathrm{M}$ has a very slight effect in the system, thus being the prediction horizon the main parameter in this case. Of course, the "Rate weight" in the "Weight tuning" tab has a significant relevance when tuning the controller for a certain desired response,

\subsection{Simulation with PID controllers (Step Input)}

The simulation results for PID controller tuning by Ziegler-Nichols \& Tyresus-Luyben methods for FOPDT model (2) obtained for flow control system shown in fig. 10, 11, and 12 respectively.

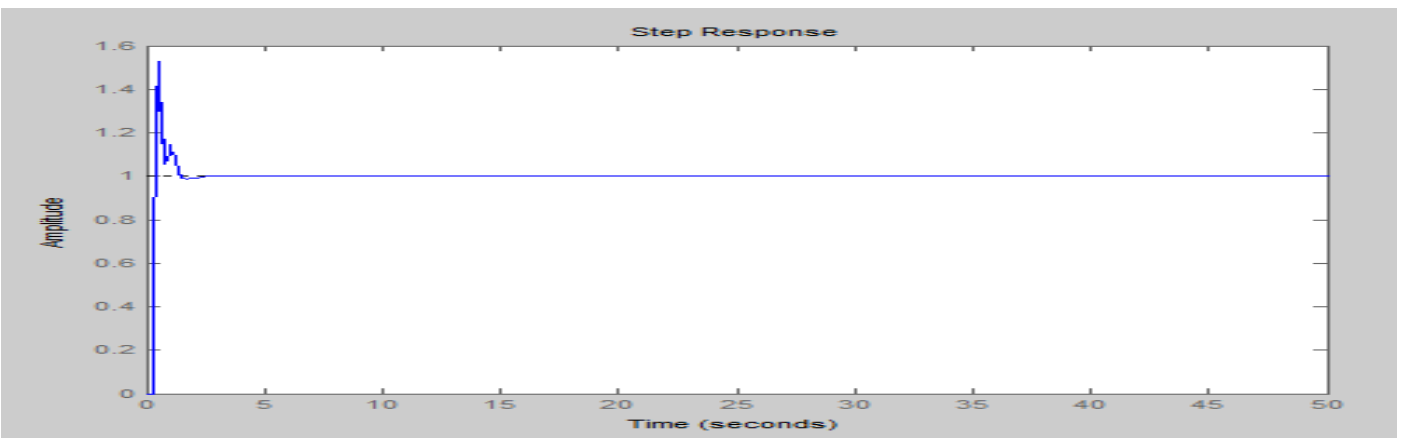

Figure 10 Unit step response of Zigler Nichols PID Controller for flow control system model (2)

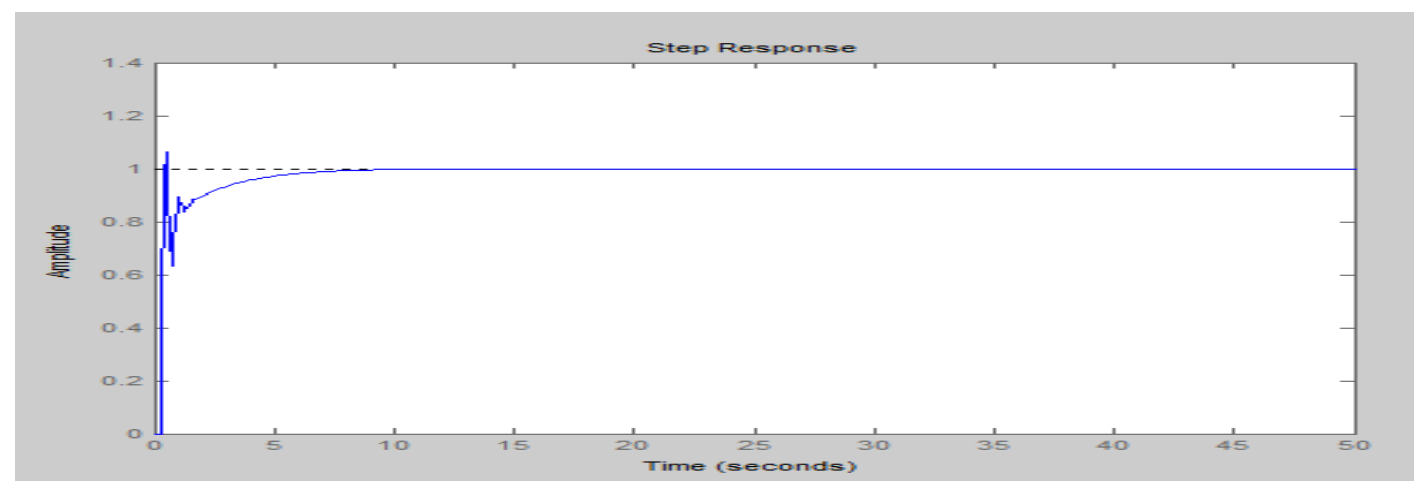

Figure 11 Unit step response of Tyreus-Luyben PID Controller for flow control system model (2) 


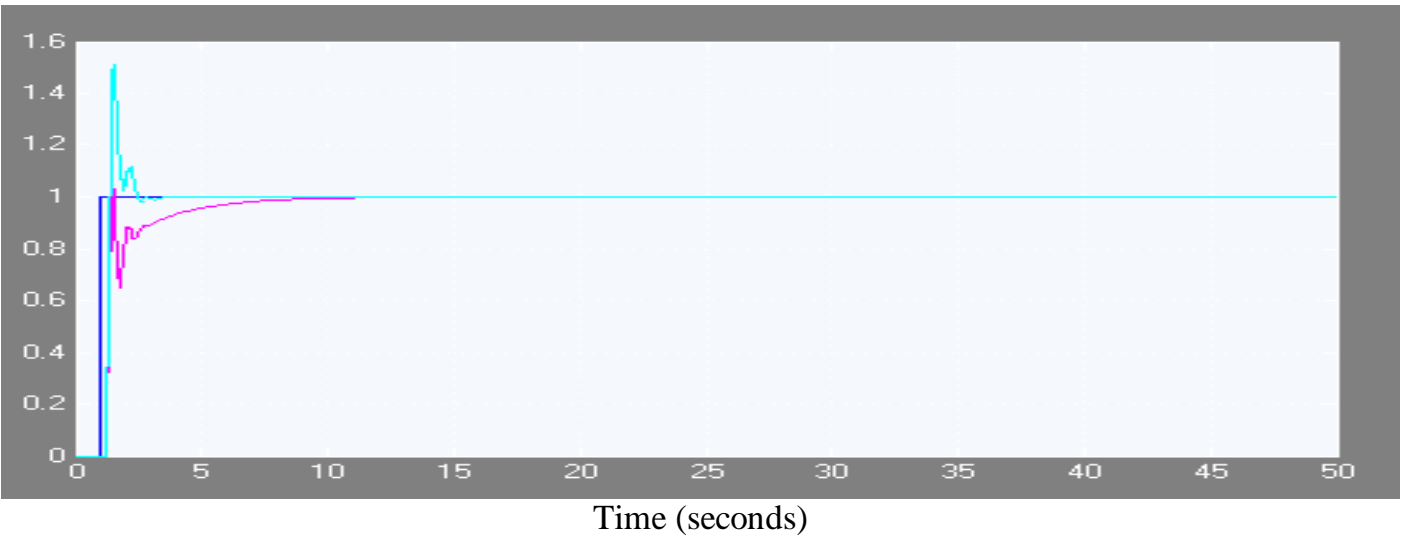

Fig 12 Combined responses of Z-N and T-L PID Controller

\subsection{Simulation with MPC controllers (Step Input)}

The step response of the proposed MPC controller with the control horizon $\mathrm{M}=3$, prediction horizon, $\mathrm{P}=17$ without manipulated variable constraint and output variable constraint is shown in Fig.13

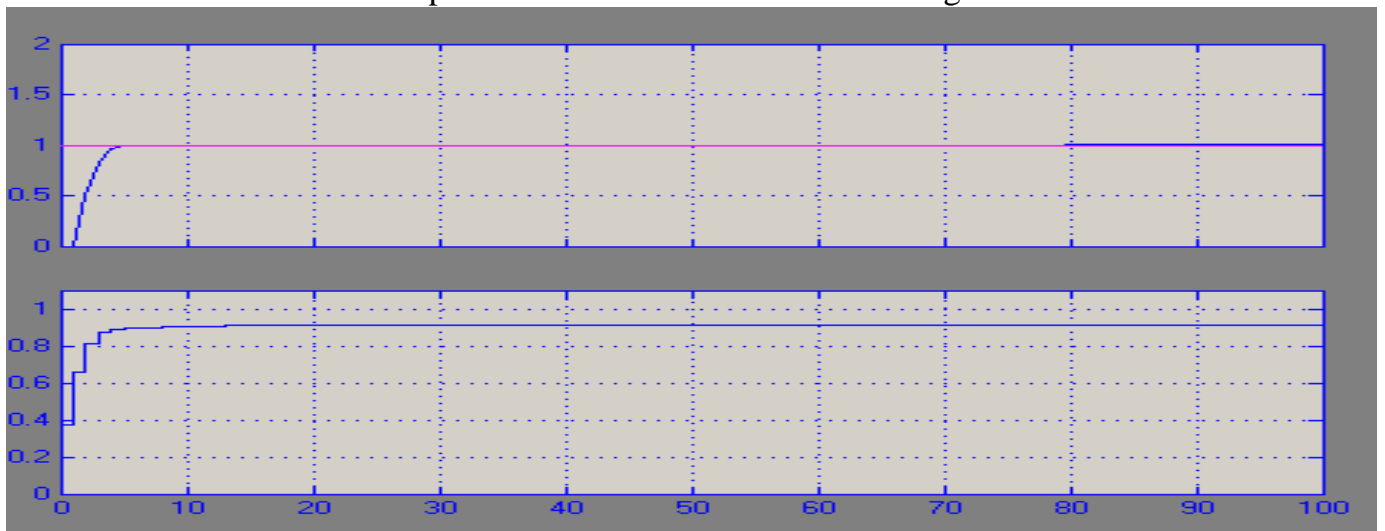

Unit step response of Model Predictive Controller for Flow control system model (2)

Table 2 shows the performance comparison results of Model Predictive control method with the conventional PID Controllers methods on the basis of time domain specifications for flow control system

\begin{tabular}{|c|c|c|c|}
\hline & $\begin{array}{c}\text { PID Controller } \\
(\mathrm{Z}-\mathrm{N})\end{array}$ & $\begin{array}{c}\text { PID Controller } \\
\text { (T-L) }\end{array}$ & MPC Controller \\
\hline Peak time (sec) & 0.13 & 0.231 & 4.75 \\
\hline Settling time(sec) & 1.62 & 8.75 & 0 \\
\hline Maximum overshoot & 48.4 & 0.993 & 0 \\
\hline Steady state error & 0 & 0 & 0 \\
\hline
\end{tabular}

From the output response of Flow control loop it was observed that MPC had almost eliminated the overshoot when compared to conventional controller which shows a more overshoot.

\subsection{Robustness analysis:}

In order to investigate the robustness of model in presence of uncertainties, the model parameters are randomly altered. For model obtained in (2), $\mathrm{K}=1.0928, t_{d}=0.248 \mathrm{sec}$ and $\tau_{P 1}=0.7699 \mathrm{sec}$. Let, these parameters are deviated as much as $20 \%$ from their nominal values due to model uncertainty. Let, there is $20 \%$ increase in dead time and gain and $20 \%$ decrease in time constant [6]

Therefore, new model is 


\section{International Journal of Innovations in Engineering and Technology (IJIET) \\ http://dx.doi.org/10.21172/ijiet.83.003}

$$
G p(s)_{2}=\frac{1.3113 e^{-0.2976 s}}{1+0.6159 s}
$$

\subsubsection{Simulation with MPC controllers (Step Input)}

The step response of the proposed MPC controller with the control horizon $\mathrm{M}=3$, prediction horizon, $\mathrm{P}=17$ without manipulated variable constraint and output variable constraint for robustness analysis of FOPDT model (3) is shown in Fig.14

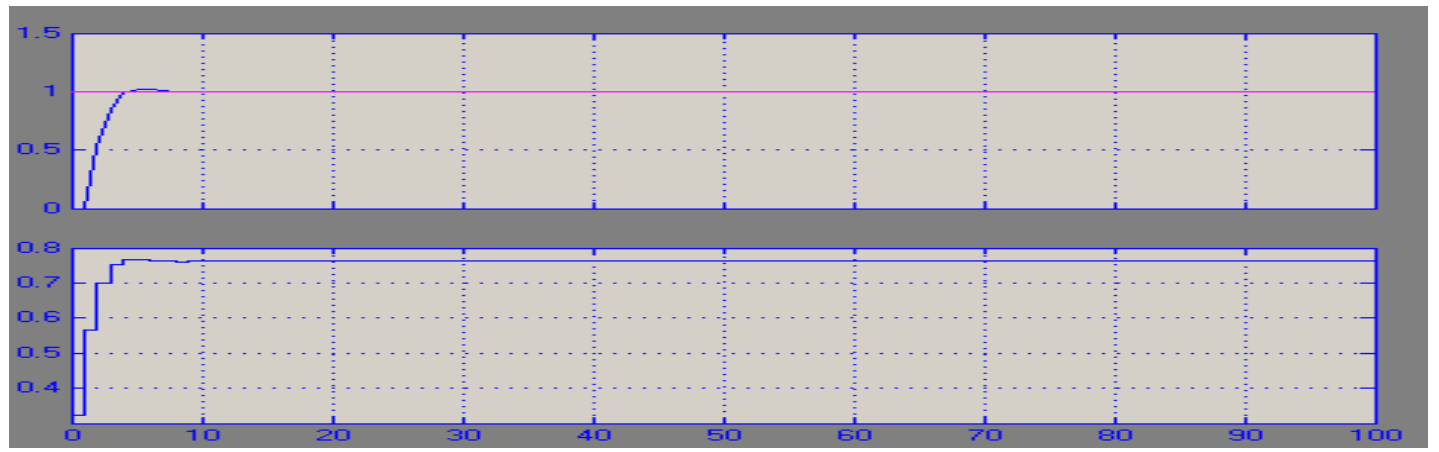

Fig no 14 Unit step response of Model Predictive Controller for Robustness analysis of flow control system model

\section{CONCLUSION}

A high performance Model based Predictive Control algorithm is proposed for the flow Control process. The MPC control algorithm is compared with conventional PID control in terms of time domain specifications like settling time, overshoot, Peak time, steady state error. The Model Predictive Controller gives better performance than PID Controller for the flow control system. MPC controller can adjust the control action before a change in the output set point actually occurs. Through simulation, the approach has been shown to be very effective for first order plus dead time processes. Compared with conventional controllers the simplified predictive controller is more robust to the process variation. Hence from the results we conclude that MPC is better than PID control.

\section{REFERENCES}

[1] R.Sivakumar, Shennes Mathew;"Design and Development of Model Predictive Controller For binary Distillation column"; International Journal of Science and Research (IJSR)(2013)

[2] S.Nithya, N.Sivakumaran, T.K.Radhakrishnan and N.Anantharaman," Soft Computing based Controllers Implementation for Nonlinear Process in Real Time", Proceedings of the World Congress on Engineering and Computer Science (2010)

[3] B.V.Anarase1, B. J. Parvat2, C. B. Kadu3, Design of Model Predictive control for non linear Process, ijetae Volume 6, Issue 1, January [2016]

[4] Ang Li M.S, Comparison between model predictive control and PID control for water- level maintenance in a two-tank system. University of Pittsburgh (2010)

[5] R.A.Darandale,C.B.Kadu and C.Y.Patil,'Design of Model Predictive Control for Temp process Proc.Of Int.Conf. on Advances in Signal Processing and Communication. [2013]

[6] J.Prakash and K.Srinivasan,"Design of nonlinear PID controller and nonlinear mode Predictive Controller for a continuous stirred tank reactor", ISA transactions, Vol.48, Issue 3, July [2009]

[7] Srinivasan K, Singh J, Anbarasan K., Paik R.,Medhi R. and Choudhury K.D, "MPC and RTDA Controller for FOPDT\&SOPDTProcess",Journal of Information Systems and communication (2012)

[8] Finn Haugen,"Comparing PI tuning methods in a Real benchmark temperature control system modeling,Identification and control, Vol.31, No. 3, [2010].

[9] A.R.Laware, V.S.Bandal, D.B.Talange. "Real Time Temperature Control System Using PID Controller an Supervisory Control and Data Acquisition System (SCADA)", in International Journal of Application or Innovation in Engineering \& Management (IJAIEM), Vol.2, Issue.2,February [2013]

[10] V.Balaji1, Dr.L.Rajaji2, comparative Study of PID and MPC Controller using Lab ViewNovember [2013]

[11] Applied Model Predictive Control A brief guide to MATLAB/Simulink ®MPCtoolbox Stefano ciannella,(2014]

[12] B.Wayne Bequette,"Process Control Modeling, Design and Simulation", Prentice Hall [2003]

[13] J.G. Ziegler and Nichols, "Optimal Settings for Automatic Controllers",Trans. ASME, vol.64 P.P. 759-768, [1942.] 